\title{
Avian nidopallium caudolaterale mediates decision-making during goal-directed navigation
}

\author{
Xinyu Liu ${ }^{1,2, *, \dagger}$, Yang Yang ${ }^{3, \dagger}$, Yanna Ping $^{1,2}$, Kun Zhao $^{4}$, Dongyun Wang ${ }^{1,2}$, Hang Xie $^{1,2, *}$ \\ ${ }^{1}$ School of Intelligent Manufacturing, Huanghuai University, 463000 Zhumadian, Henan, China \\ ${ }^{2}$ Henan Engineering Reseacrh Center of Intelligent Human-Machine Interaction Equipment, Huanghuai University, 463000 Zhumadian, Henan, China \\ ${ }^{3}$ Department of Neurosurgery, Zhumadian Central Hospital, 463000 Zhumadian, Henan, China \\ ${ }^{4}$ School of Electrical Engineering, Zhengzhou University, 450001 Zhengzhou, Henan, China
}

*Correspondence: liuxinyu@huanghuai.edu.cn (Xinyu Liu); xiehang199228@hrbeu.edu.cn (Hang Xie)

$\dagger$ These authors contributed equally.

DOI:10.31083/j.jin2004095

This is an open access article under the CC BY 4.0 license (https://creativecommons.org/licenses/by/4.0/).

Submitted: 3 August 2021 Revised: 7 November 2021 Accepted: 26 November 2021 Published: 30 December 2021

Previous work demonstrates that nidopallium caudolaterale, which is considered to be an analog of the mammalian prefrontal cortex, participates in goal-directed navigation in pigeons. However, its role remains unclear. To clarify its role, two goal-directed navigation tasks in plus-maze were designed, in which the goal location of one is random, and the other is fixed, i.e., the random-goal task and the fixed-goal task. The animals were trained to run from the starting location to the goal location in accordance with the cue in the plusmaze. The goal location is variable for the random-goal task but unchanged for the fixed-goal task. The results have demonstrated that the time point of nidopallium caudolaterale neuron response is consistent with decision-making. During the decision-making, the firing rates significantly increased in two tasks, which can also decode the direction of upcoming movement in the random-goal task. However, the location of decision-making is different between the tasks mentioned above. The decision-making window is at the intersection in the random-goal task, which is a departure in the fixed-goal task. In addition, these results also provide evidence that the neural activities obtained from the nidopallium caudolaterale may contain the decision-making information during goal-directed navigation. These results suggest that the avian nidopallium caudolaterale and the mammalian prefrontal cortex may play a similar role in goaldirected spatial decision-making. Additionally, these also may provide some support to understand the neural mechanism of decisionmaking for different species.

\section{Keywords}

Pigeons; Nidopallium Caudolaterale; Decision-making; Coal-directed navigation; Prefrontal cortex

\section{Introduction}

Nearly all animals can navigate to the desired location by using information about environmental landmarks and their movements in nature. The behavior divined by a specific goal is often goal-directed navigation [1]. Goal-directed navigation is a complex behavior that requires the subject to perceive its environment, learn about its significance, and then select where to go next based upon what has already been learned. Rodent experimental evidence highlights the central role of the prefrontal cortex (PFC) in this process [2-5]. As the recipient of input from several structures involved in learning and memory, the PFC is well placed on integrating affective information to produce adaptive behavior [4] Hok et al. [6] reported that PFC neurons expressed sustained activity in a goal-directed navigation task at the goal zone. More importantly, lesions of the PFC cause impairments in goal-directed behavior [7]. Nevertheless, as the analog of the mammalian PFC, the role of avian nidopallium caudolaterale (NCL) in goal-directed navigation remains unclear.

The NCL is a multimodal associative forebrain area that receives input from secondary sensory areas of all modalities and projects to both limbic and sensorimotor striatum and premotor areas [8]. Accumulating evidence strongly proves the functional equivalence of the NCL and the PFC with regards to neurochemistry [9], anatomy [10], behavior [11], and electrophysiology $[12,13]$. Previously, we found the firing rates of the NCL neurons significantly increased during the decision-making process, which is associated with the choice of the upcoming movement [14]. In addition, our further research has also found that the information flow from the hippocampus ( $\mathrm{Hp})$ to the NCL exists during the avian goal-directed decision-making [15]. These studies suggest that the activities of the NCL neurons may be related to the decision-making in goal-directed navigation.

To help clarify the role of NCL in goal-directed navigation, two goal-directed navigation tasks in plus-maze were designed, in which pigeons were trained to go back and forth between the starting location and the goal location. The difference between the two tasks refers to that the goal location in one task is random, in which the pigeons must choose one of three directions according to the cue in the goal location, and it is fixed in the other task, i.e., the goal location is not changed in the whole experiments. The neural activities were recorded from the NCL when pigeons performed these 
A
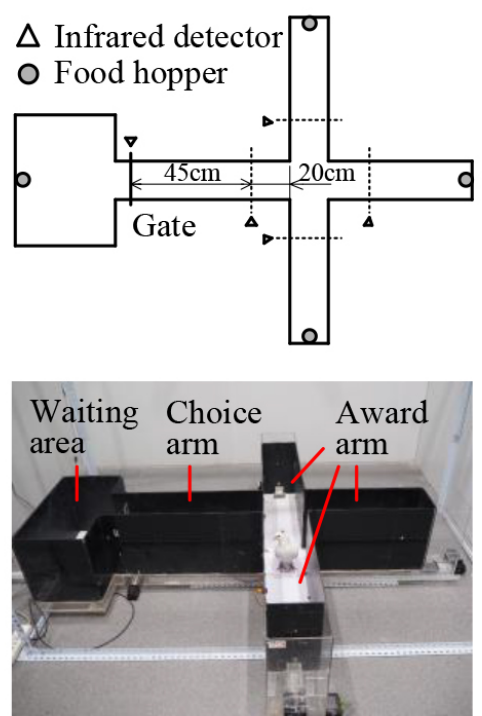

D

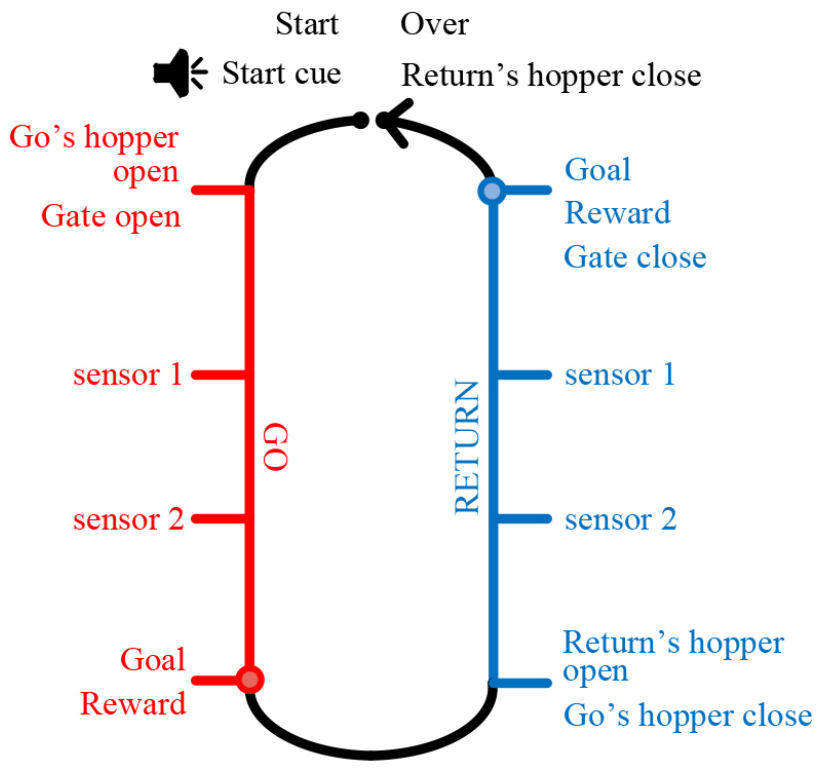

B

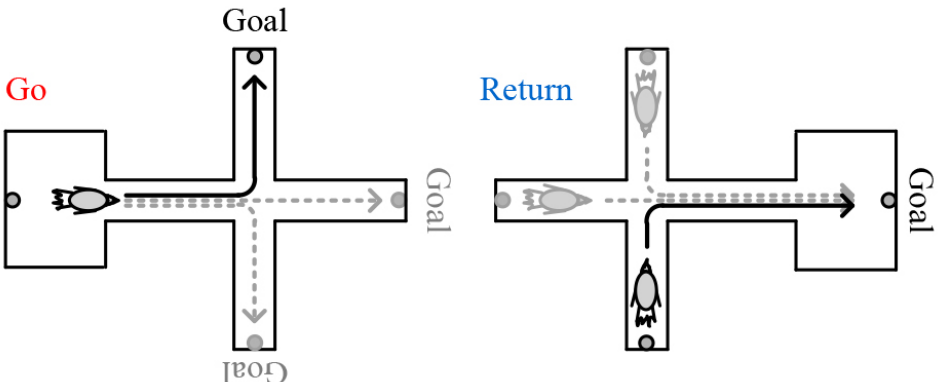

C
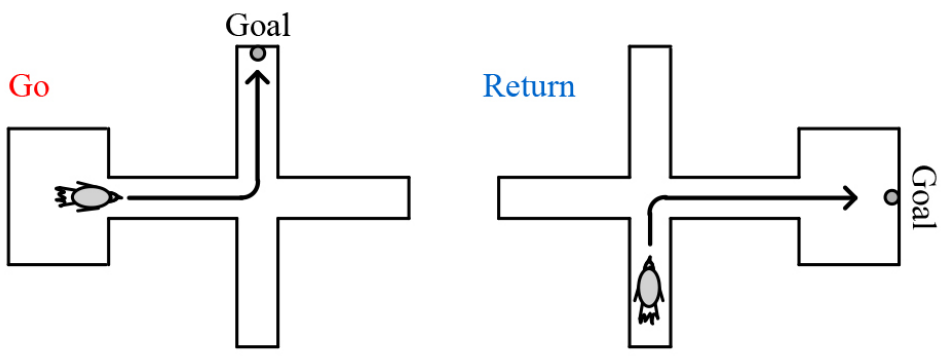

$E$

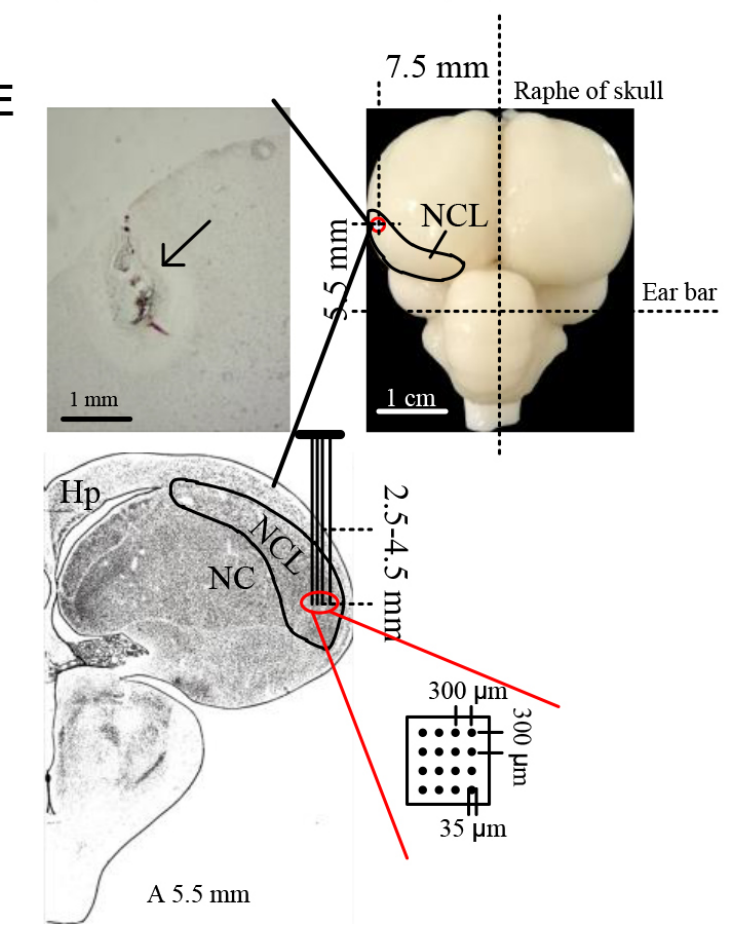

Fig. 1. Behavioral task and experimental procedures. (A) Diagram of experimental apparatus for pigeon. The infrared detector on the choice arm was defined as sensor 1, an infrared detector on the award arm was defined as sensor 2. (B) Diagram of the random-goal task. (C) Diagram of the fixed-goal task. (D) Experimental procedure. The red line represents going, and the blue line denotes returning. The arrow represents the direction of movement. (E) Implanting location of microelectrode array, which is denoted by red circle.

two tasks. The neural activities were analyzed and compared in the going and returning tasks with random goal locations. Meanwhile, the NCL neural activities in the task with fixed goal location were also studied. Our paper aims to clarify the role of NCL during goal-directed navigation.

\section{Materials and methods}

Many of the procedures used here have been described previously. For further details, please refer to Liu et al. [14]. Only a brief description of the major steps is provided. All experiments were conducted in line with the Animals Act, 2006 (China) for the care and use of laboratory animals and approved by the Life Science Ethical Review Committee of Huanghuai University (No. LL2020001).

\subsection{Subjects}

Ten adult pigeons (Columba livia, weighing 450-550 g, sex unknown) were housed in individual wire-mesh cages within a colony room. During the experiment, water was available, but the food was restricted to daily testing on workdays, with 
A
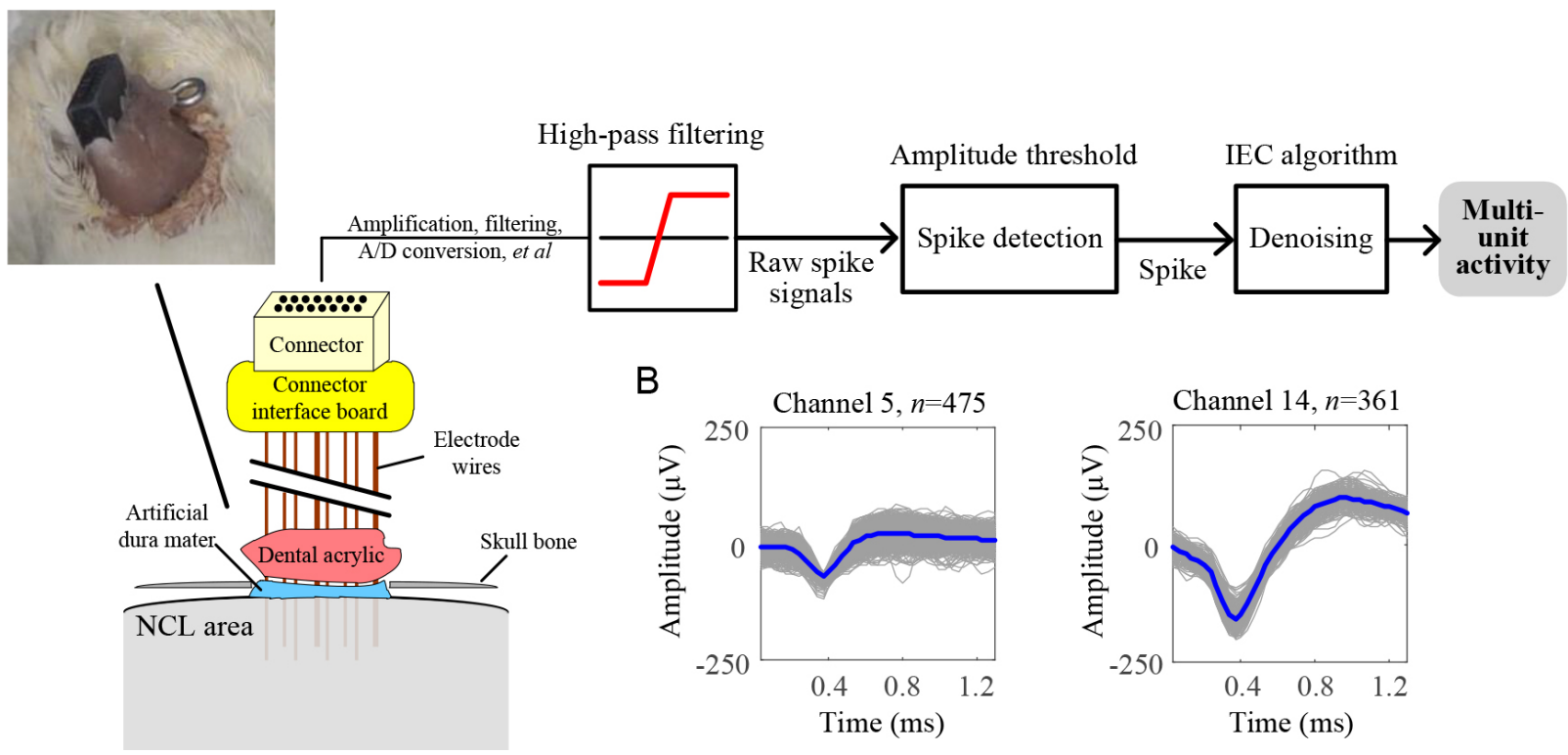

Fig. 2. Data acquisition and processing. (A) Data acquisition and processing flow. A photograph of the implanted microelectrode array is shown in the upper left corner. (B) Spike waveforms of two typical channels after processing. The mean spike waveform is highlighted in blue.

additional free food available on weekends. To distinguish, each pigeon was numbered by P010, P600, P601, P605, P609, P619, P101, P104, P109, and P111, respectively. The first six pigeons performed the goal-directed navigation task with random goal location (named the random-goal task). Besides, the last four performed the goal-directed navigation task with fixed goal location (named the fixed-goal task). Only one session was performed in one day for each animal, including 30 trials.

\subsection{Behavioral apparatus and protocol}

Pigeons were trained to perform a goal-directed navigation task for food rewards in a plus-maze, a cross-shaped box with four arms at angles of 90 degrees. Four automatic food boxes were located at the ends of each arm. Five infrared sensors and four arms were located at the gate to detect when a pigeon passed the specific locations. The infrared sensor on the choice arm is defined as sensor 1, and that on the award arm is defined as sensor 2. The detail of the plus-maze is illustrated in Fig. 1A.

For the random-goal task, pigeons were trained to turn left, move forward, or turn right at the intersection according to the cues. The sequence of directions is a pseudo-random sequence set in the program to ensure that the number of occurrences in all directions is the same. The cues are the food and light at the end of the reward arm. After the reward is consumed, it must return to the starting location. This task includes two processes, the going and returning (see Fig. 1B). The animal needs to decide at the intersection according to the cue because the goal location was random. However, the goal location is fixed during returning, and the pigeon already knows the goal location before departure. Thus, the returning is also a fixed-goal task (see Fig. 1C). The NCL neural activities between the going and returning were first compared. Then, those going between the random-goal and the fixed-goal tasks were compared.

The training is divided into two phases to reduce the impact of surgery, i.e., the pre-training phase and the training phase. The pre-training phase mainly aims to familiarize the animals with the experimental environment and procedures, generally lasting 2-3 days. In this phase, pigeons that are easily fly, timid and not susceptible to food will be eliminated. Following pre-training, pigeons were implanted with recording electrodes. After 5-7 days of recovery, the training phase was performed until recordable neurons were found. After a week, the pigeons could learn the behavior easily and became completely trained. The experiment procedure is shown in Fig. 1D.

\subsection{Surgery and recordings}

All surgeries were performed while animals were under general anesthesia with $3 \%$ pelltobarbitalum natricum $(0.12$ $\mathrm{mL} / 100 \mathrm{~g}$ body weight). The data was collected by employing self-made 8 or 16 channel platinum-iridium microwire arrays $(4 \times 4$ or $4 \times 2$, California Fine Wire Company, Grover Beach, USA), which was chronically implanted in the left and/or right NCL area (see Fig. 1(E); AP: $5.5 \mathrm{~mm}$, ML: \pm 7.5 $\mathrm{mm}$, DV: $2.5-4.5 \mathrm{~mm}$ ) based on the atlas provided by Karten and Hodos [16].

Neural signals were recorded with a Cerebus ${ }^{T M}$ recording system (Blackrock Microsystem Inc., Salt Lake City, USA). The signals were amplified $(4000 \times)$, filtered $(0.25-5$ $\mathrm{kHz}$ ) and continuously sampled at $30 \mathrm{kHz}$, and subsequently detected unidirectional crossings of an amplitude threshold (4-5 times root mean square value of the filtered signals) as spikes. Spikes were denoised by the inter-electrode corre- 
A
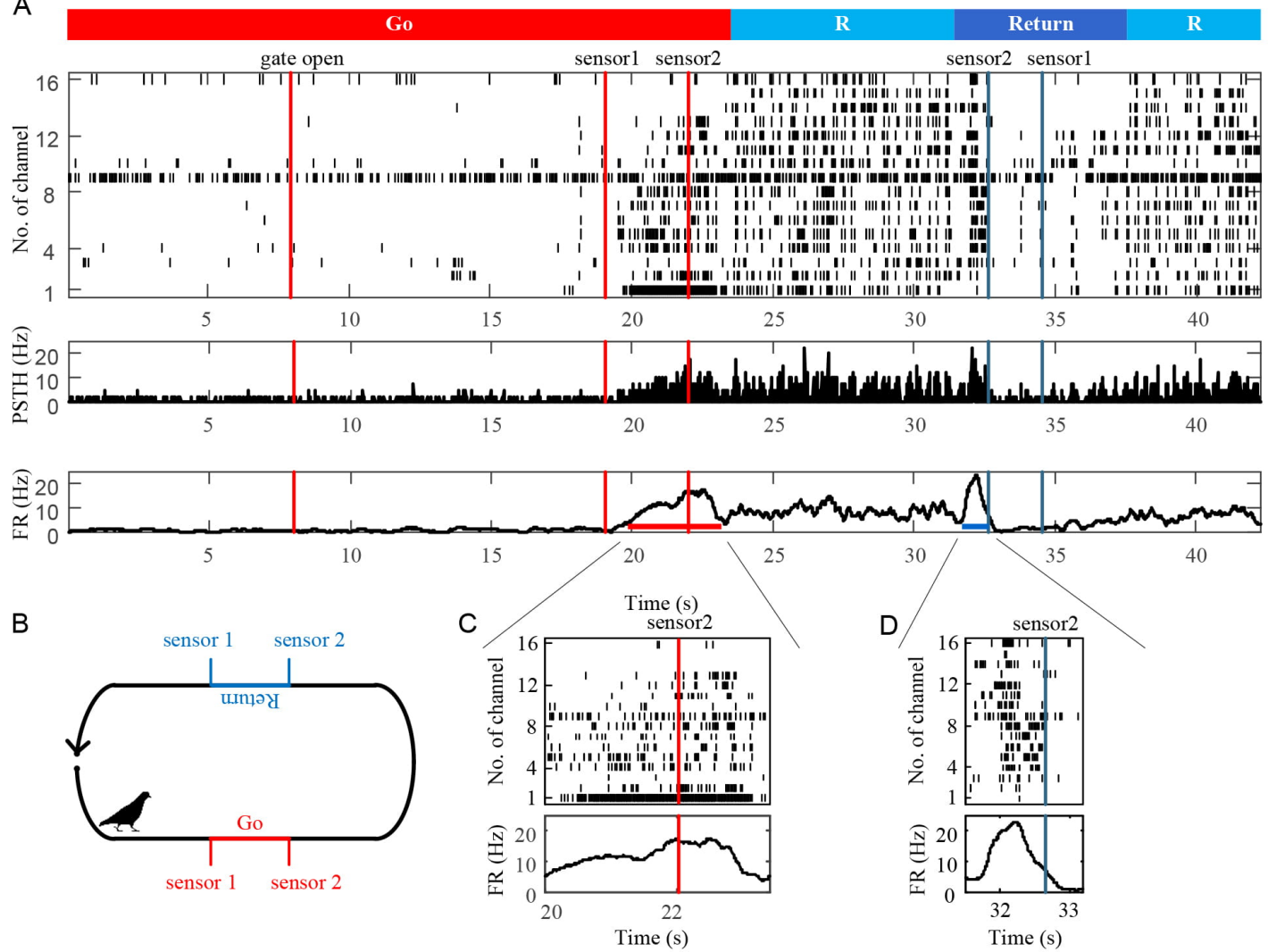

$\mathrm{E}$
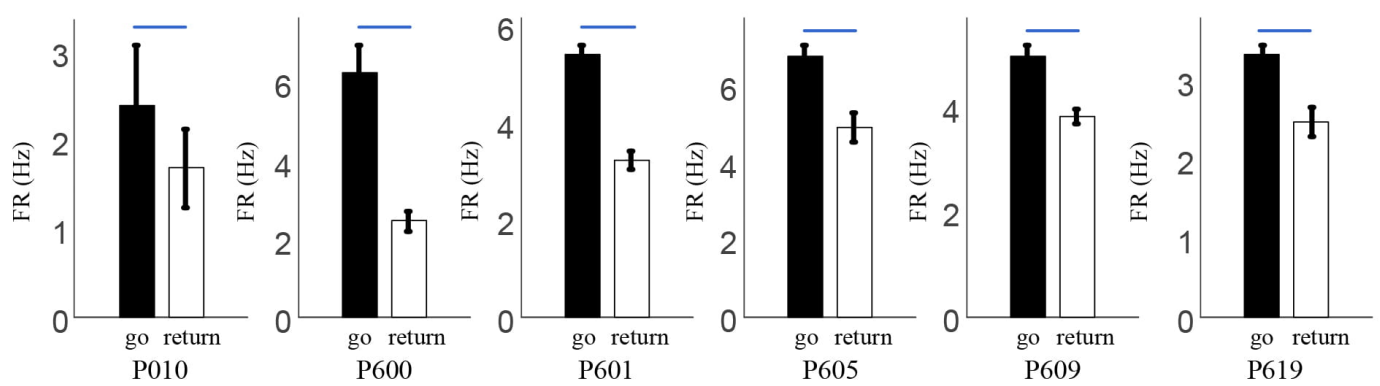

$\mathrm{F}$
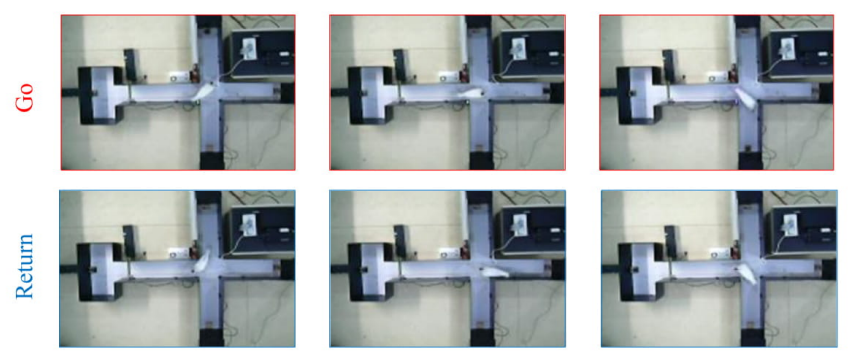

G

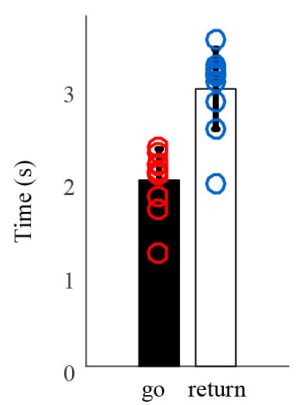

Fig. 3. NCL neural activities in the random-goal task. (A) Example of neural activity in a trial. From top to bottom: illustration of the different event windows, spike trains, peri-event time histogram (PSTH), and firing rate (FR) curve. The event window is marked with a colored rectangle. R: food rewards. A dot raster shows a spike for spike trains. The firing-rate curve is obtained by averaging the dot raster and smoothing it with a $0.1 \mathrm{~s}$ boxcar window. Vertical color lines indicate when the pigeon passes the corresponding infrared detector. Horizontal color lines highlight the response of neurons. (B) Diagram of decision-making window, which is marked with the color lines. (C) and (D) Enlarged view of the detail, marked in the horizontal line in (A). (E) Comparison of firing rate between going and returning at the intersection is marked in (B). (F) Behavioral photographs of the pigeon at the intersection of the maze. Top: photographs of the turning in going. Bottom: photographs of the turning in returning. (G) Comparison of the duration for the decision-making between going and returning. The circle denotes the mean value of all trials for a pigeon, in which the red circle represents the going, and the blue circle denotes the returning. Error bars indicate standard error of mean (SEM). 


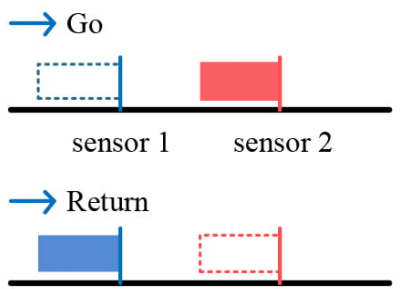

sensor 2 sensor 1

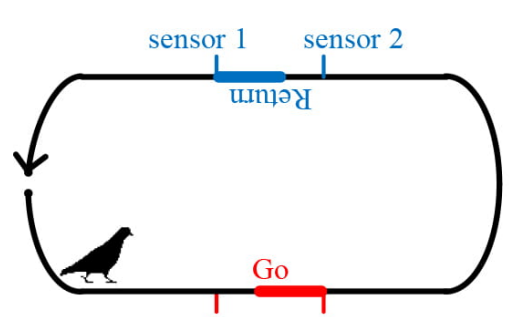

sensor 1 sensor 2

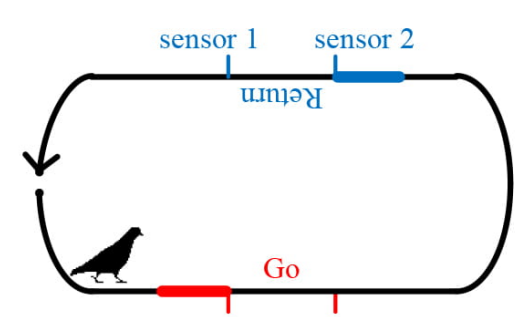

sensor 1 sensor 2
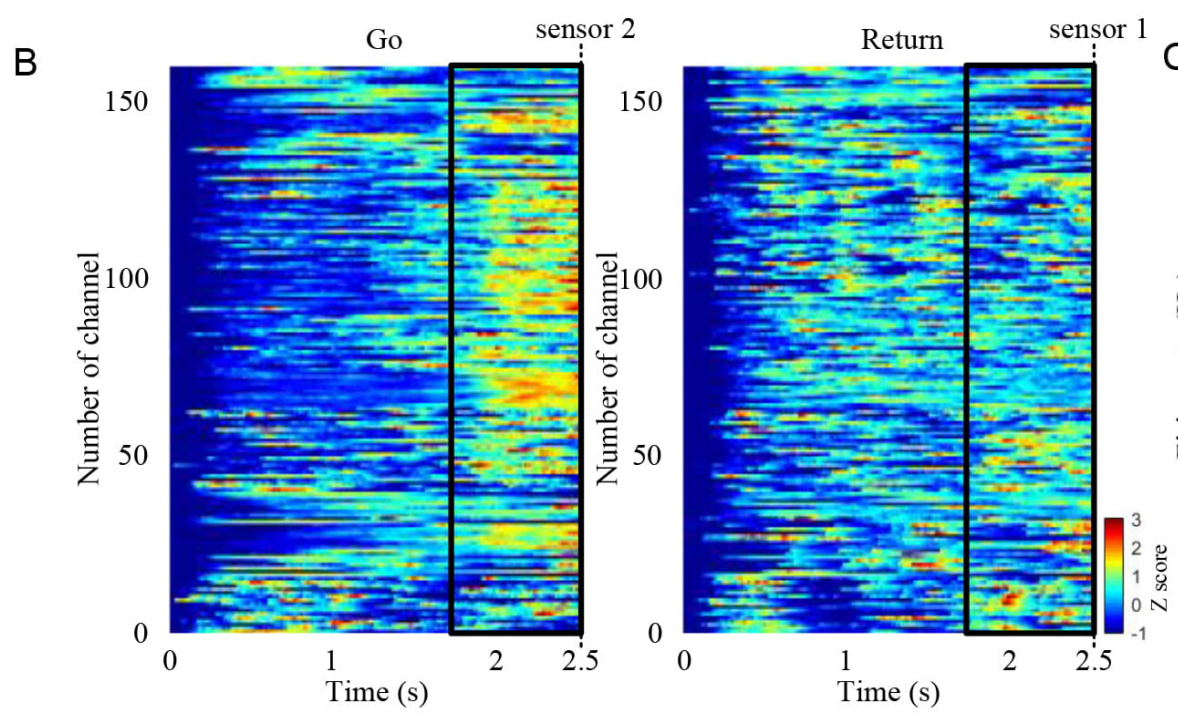

C
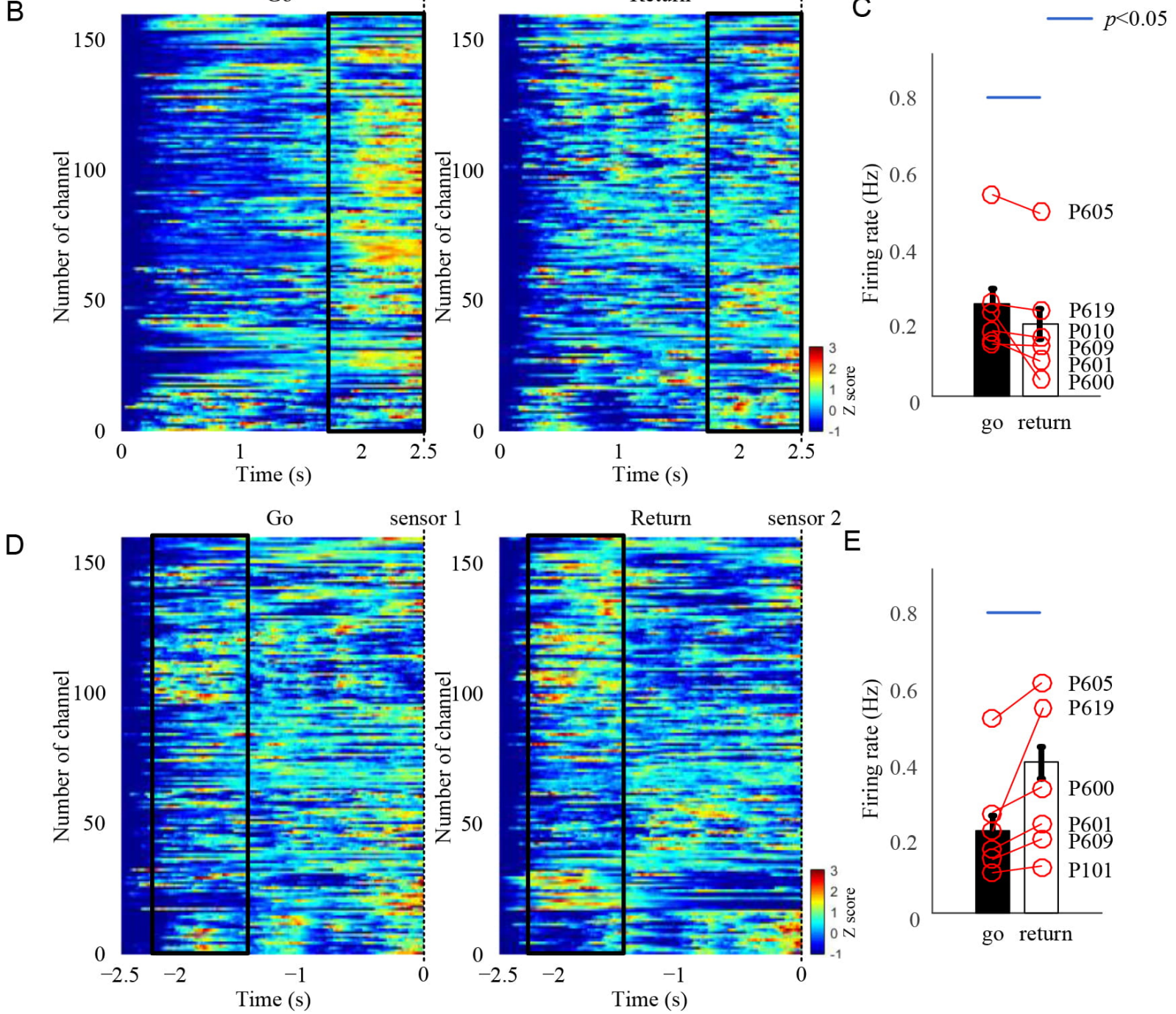

Fig. 4. NCL neural activities include decision-making information. (A) Diagram of the location of decision-making. Left: the location of decisionmaking is shaded by a rectangle, and the dotted line is the relative location of the decision-making window. Middle and Right: diagram of the location of the window (2.5 s) for calculating the firing rate in (C) and (E), respectively, which is shaded with the color lines. (B) and (D) The average, $Z$-scored activity of a single task-modulated channel. Time is measured from sensor 1 in the going (left) and sensor 2 in the returning (right). Individual channels $(n=160)$ are numbered on the y-axis. Colors depict instantaneous neural firing rates (0.1-s bins) averaged across all trails. The black boxes highlight their differences. (C) and (E) Comparison of firing rate between going and returning in different windows. The data was normalized by its maximum value. The (C) conforms to the middle, and the (D) corresponds to the right sub-figure in (A). Each red line denotes a pigeon. Error bars indicate SEM.

lation technique [17]. After denoising, spikes from the same channel were defined as the multi-unit activity. The data processing flow and two typical spike waveforms are presented in Fig. 2.
After completing all the recordings, the tips of recording electrodes in the NCL were marked by electrolytic lesions (5 $\mathrm{mA}$ for $30 \mathrm{~s}$ ) [14]. After heart perfusion, the brain was fixed in formalin sucrose solution for $48 \mathrm{~h}$. Besides, the frozen 
A

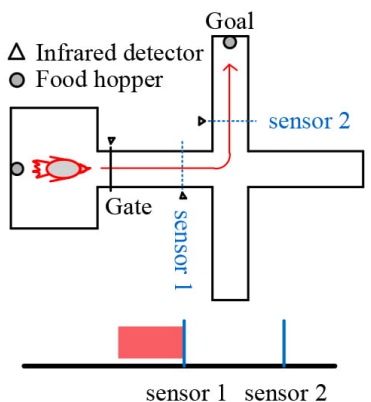

B

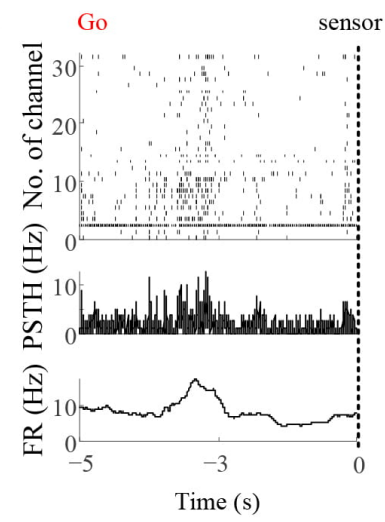

D

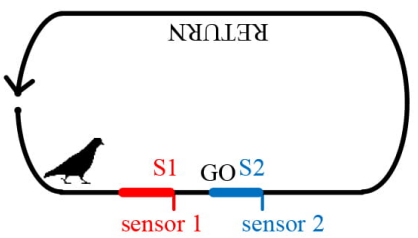

C
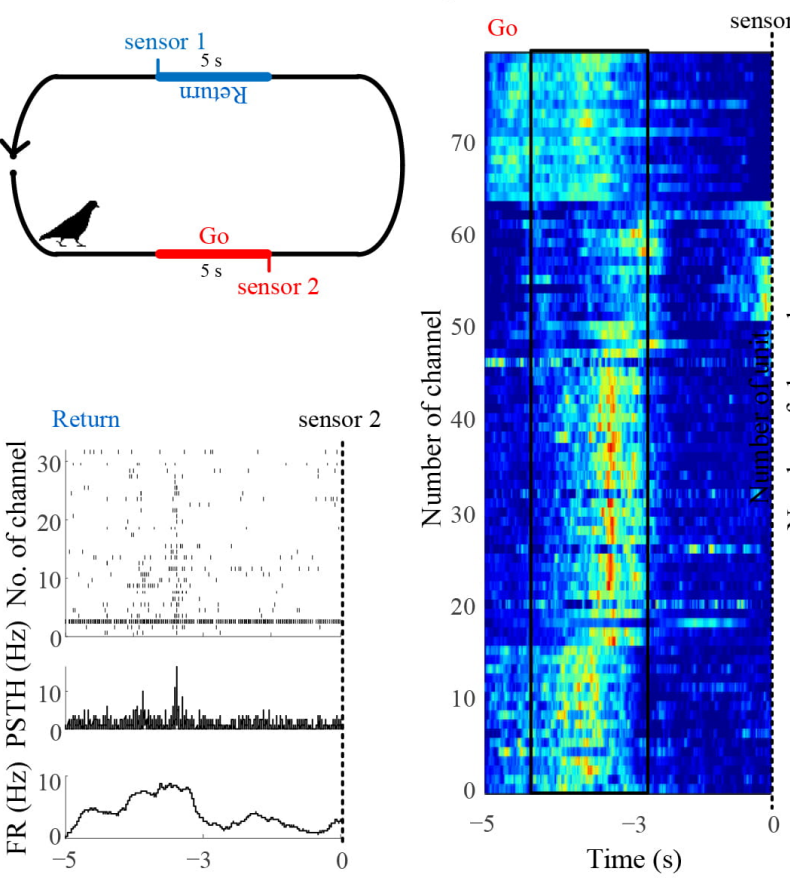

1 Return

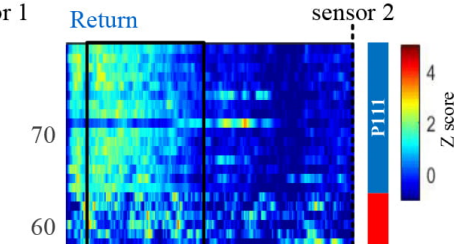

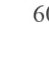

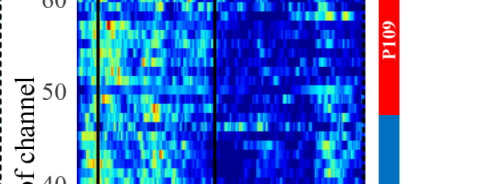

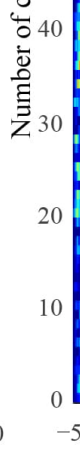

$\begin{array}{ccc}-5 & -3 & 0 \\ & \text { Time }(\mathrm{s}) & \end{array}$

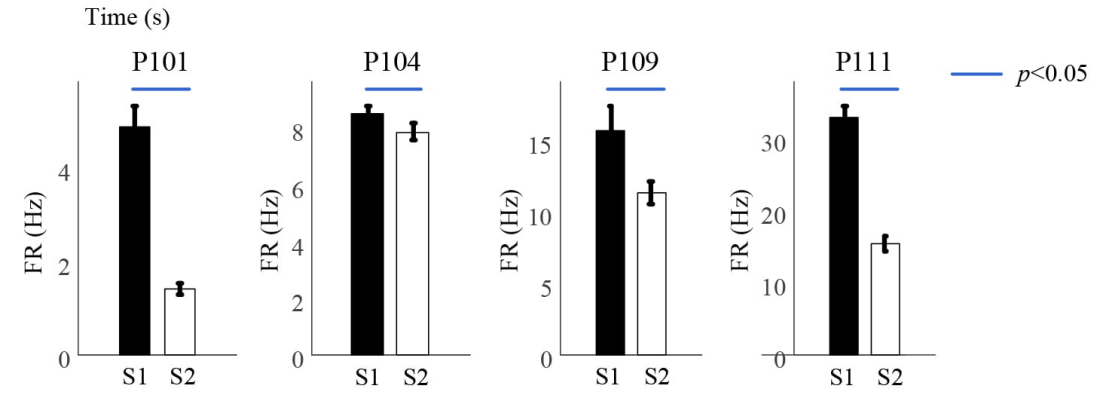

Fig. 5. NCL neural activities in the fixed-goal task. (A) Diagram of the task (top) and the location of decision-making (bottom), and diagram of the event window (right) for (B) and (C). (B) Examples of neural activities at the intersection in a trial are highlighted in (A). Dashed lines indicate the location of the sensor. From top to bottom: raster plots, PSTH, FR curve obtained by averaging the dot raster and smoothing with a $0.1 \mathrm{~s}$ boxcar window. (C) The average, Z-scored activity of single task-modulated channels across all trials $(n=80)$. Dashed lines indicate the location of the sensor. Colors depict instantaneous neuronal firing rates (0.1-s bins), averaged across all animal trails. (D) Comparison of the firing rate between the S1 and S2 windows. S1 is expressed as $2.5 \mathrm{~s}$ before sensor 1, and S2 is defined as $2.5 \mathrm{~s}$ before sensor 2, as shown in the left sub-figure. Error bars indicate SEM.

coronal sections $(50 \mu \mathrm{m})$ were collected from the recording sites. The locations of the recording electrodes in the brain were histologically examined (see Fig. 1E. left).

\subsection{Data analysis and statistics}

Only analyzes the test data with the completion time $<60$ s. All analyses were performed using MATLAB software (r2016a, Mathworks Inc., Natick, USA). In terms of the behavioral data, the time taken by the decision-making at the intersection, which was from sensor 1 to sensor 2 in going and from sensor 2 to sensor 1 in returning, was calculated. Regarding the electrophysiological data, a trial was considered usable if it fulfilled the following two criteria: (1) the trial contains a complete experimental process and (2) the time taken by decision-making is less than $10 \mathrm{~s}$ in going. After data screening, in the random-goal task, there are 90 trials for P010, 141 trials for P600, 47 trials for P601, 51 trials for
P605, 102 trials for P609 and 73 trials for P601. In the fixedgoal task, there are 120 trials for P101, 49 trials for P104, 367 trials for P109 and 196 trials for P111. Unless otherwise specified, the data in text and figures was expressed as mean \pm standard deviation, which was assessed by employing a bootstrap method [18], a computer-based method that depends on random sampling with replacement.

For the random-goal task, the movement direction of the animal was decoded in each trial using the firing rates of all channels recorded in it. Leave-one-output decoding was performed with a $k$-nearest neighbor method. According to our previous research results, $k$ is set to 1 [19]. For each trial, the event type was predicted based on the distribution of all the remaining trials. In other words, a trial was selected as the test dataset and other trials as the training dataset. Besides, the training dataset was labeled the class. Then, the 

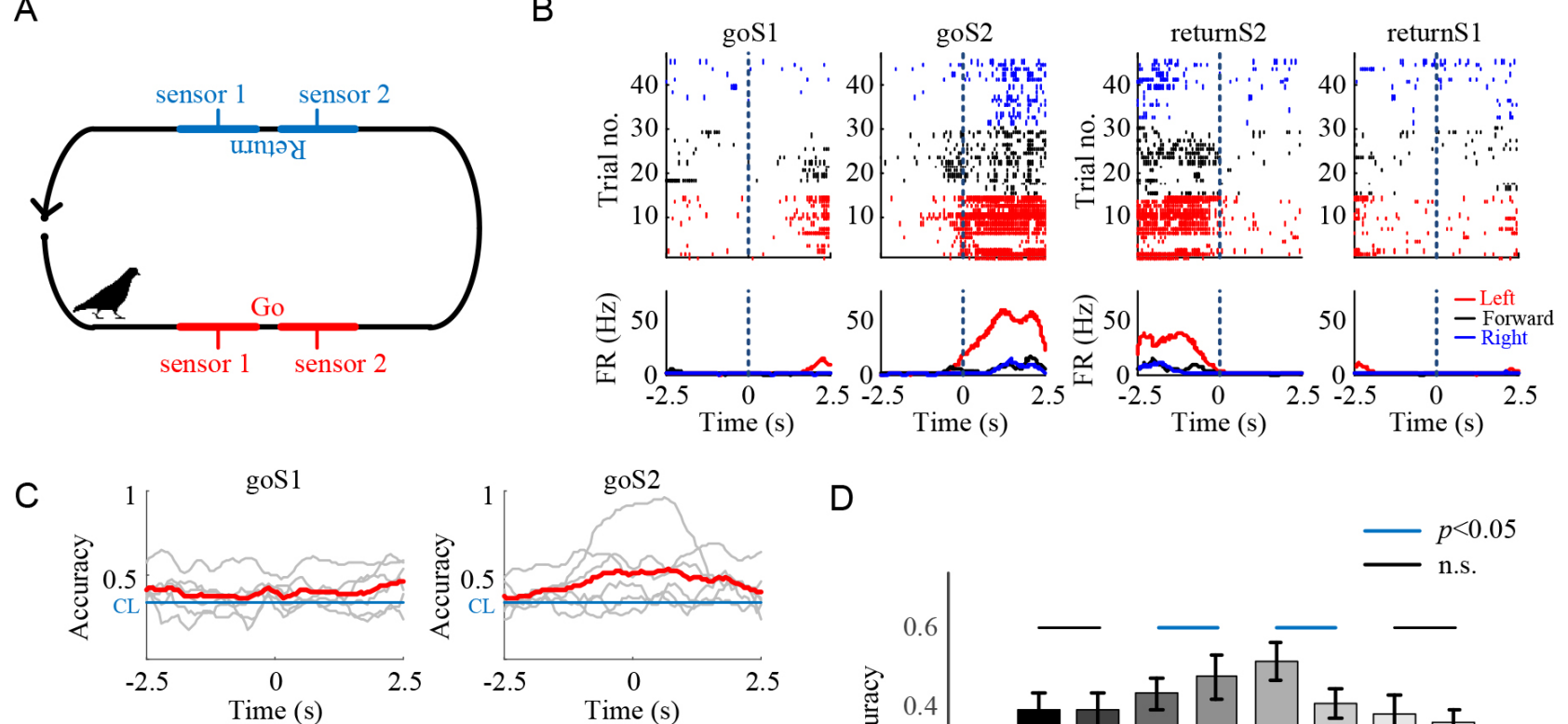

D

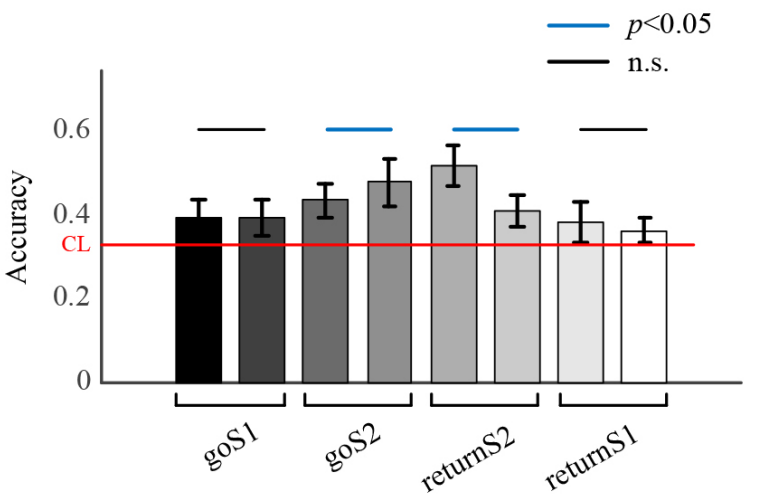

Fig. 6. Decoding performance of the NCL neural activities during the decision-making process. (A) Diagram of the event windows ( $5 \mathrm{~s}$ ), marked with colored lines, centered at the task events. The event windows are defined respectively as goS1, goS2, returnS2, and returnS1 in accordance with chronological order. (B) Spike train raster from a recorded channel in the different event windows. Top: spike trains for all trials. A train denotes a trial. A dot raster represents a spike. Bottom: the firing rate curve was obtained by averaging the dot raster and smoothing it with a $0.2 \mathrm{~s}$ boxcar window. (C) Dynamic accuracy in different event windows. Time is measured from sensor 1 and sensor 2 for going and returning. The red line is mean, and the blue is chance level (CL, 0.33). Each gray line denotes an animal. Note that the accuracy near time 0 is significantly higher than the chance level in goS2 and returnS2. (D) Comparison of accuracy among different event windows. For each event window, the left bar is accurate before time 0 , and the right bar is accurate after time 0 . The red line is the CL $(\sim 0.33)$. Error bars indicate SEM.

class of the test dataset was predicted by the $k$-nearest neighbor method. Repeating the step until the class of all trials was predicted. Finally, the predicted labels and the real labels were compared. Meanwhile, the accuracy is calculated as the percentage of correctly classified trials.

For the statistical analysis, statistical differences were evaluated by performing a Wilcoxon signed-rank test, and the significance level was set to $5 \%$, i.e., $p<0.05$ was considered significant. The Wilcoxon signed-rank test is a rank-based alternative to the parametric $t$-test that assumes only that the distribution of differences within pairs is symmetric without requiring normality [20]. The permutation test assessed the statistical significance of decoding accuracy, which compares the observed data to data with shuffled labels to test the null hypothesis that the decoding accuracy is obtained by chance [21]. The significance was set at 0.05 for the permutation test $(p<0.05)$.

\section{Results}

\subsection{NCL neural activities in the goal-directed navigation task}

In general, 160 channels were recorded for the randomgoal task, 16 channels for P010, 16 channels for P600, 32 channels for P601, 32 channels for P605, 32 channels for P609, and 32 channels for P619. In the plus-maze, the natural characteristics of intersection, where animals must make a decision, make it an intuitive candidate window for the decision-making. As a result, the neural activities from the NCL were analyzed at the intersection. An example of spike trains from a full trial was presented in Fig. 3A. The results have demonstrated that the neural activities between the going and returning are different at the intersection. During the going, the firing rates increased as pigeons reached the intersection and peaked near sensor 2 (see Fig. 3C). Comparatively, the firing rates increased during the returning as pigeons left the goal location and peaked before sensor 2 (as shown in Fig. 3D). Unlike in the going, the firing rates did not increase significantly at the intersection in the returning. 
In addition, we compared both the firing rates and the behavioral data between going and returning at the intersection. The window was defined as how the animal passed from sensor 1 to sensor 2 (see Fig. 3B). The firing rates in the going are significantly higher than that in the returning for all pigeons (Wilcoxon signed-rank test, $p<0.05$, $n=6$ ) (see Fig. 3E). Collectively, these results have shown that the decision-making window in the going and returning should be different. The decision-making window in the going should be at the intersection because pigeon can only determine the goal location when it reaches the intersection and find the cue. The decision-making window in the returning should be before sensor 2 because the animals already know the goal location before departure. Afterward, we compared the time spent in the decision-making window between the going and returning for all trials (see Fig. 3F). Although the time of returning $(3.00 \pm 0.3 \mathrm{~s})$ is higher than that of the going $(2.15 \pm 0.3 \mathrm{~s})$ for most pigeons (see Fig. $3 \mathrm{G})$, they remain around $2.5 \mathrm{~s}$.

\subsection{NCL neural responses during the decision-making}

According to the above analysis, it is known that the decision-making window is at the intersection for the going, and it appears before sensor 2 for the returning. The neural activities in these two windows were explored (see Fig. 4A left). Specifically, the neural activities from $2.5 \mathrm{~s}$ before the sensor 2 to the sensor 2 in the going and from $2.5 \mathrm{~s}$ before the sensor 1 to the sensor 1 in the returning (see Fig. 4A middle) as well as from $2.5 \mathrm{~s}$ before the sensor 1 to the sensor 1 in the going and from $2.5 \mathrm{~s}$ before the sensor 2 to the sensor 2 in the returning were used (see Fig. 4A right). The selection of 2.5 $\mathrm{s}$ time lengths is because the length of the turning window is generally about $2.5 \mathrm{~s}$. It is hoped that the window can cover the turning window.

Based on a comparative analysis of neural activity between the going and returning in the two windows, the firing rates have increased significantly near sensor 2 in both going and returning for the most channels (see Fig. 4B and Fig. 4D; $n$ $=160$ ). It should be pointed out that the animal first passes through sensor 1 in the going and sensor 2 in the returning. Therefore, the neural activities were aligned at sensor 1 in the going and sensor 2 in the returning. Fig. 4B shows 2.5 $\mathrm{s}$ before sensor 2 in the going (left) and sensor 1 in the returning (right). Fig. 4D presents $2.5 \mathrm{~s}$ before sensor 1 in the going (left) and sensor 2 in the returning (right). Moreover, the firing rates in the window before sensor 2 in the going are significantly higher than those in the window before sensor 1 in the returning (see Fig. 4C, Wilcoxon signed-rank test, $p<0.05, n=6$ ). In contrast, the firing rates in the window before sensor 2 in the returning are significantly higher than those in the window before sensor 1 in the going (see Fig. 4E, Wilcoxon signed-rank test, $p<0.05, n=6$ ). These results have shown that the NCL neural activities may be related to the decision-making in goal-directed navigation.

In the above goal-directed navigation task, the going is a random-goal task, and the returning is a fixed-goal task. As a result, a new fixed-goal task was performed in the same plusmaze using 4 pigeons to further validate the above results. Generally, 80 channels were recorded (16 channels for P101, 32 channels for P104, 16 channels for P109, and 16 channels for P111). Similar to the returning in the above goal-directed navigation task, the decision-making should also occur before the intersection during the new task (see Fig. 5A). The view can be confirmed by an example trial in the going and returning (see Fig. 5B). The firing rates' peak of most channels appears near sensor 1 for the going and sensor 2 for the returning (see Fig. 5C). To compare with the results of the random-goal task, we aligned the neural activities in Fig. 5C at the same location with the neural activities presented in Fig. 4B and D. According to the obtained result, we compared the firing rates between the window $2.5 \mathrm{~s}$ before sensor 1 and the window $2.5 \mathrm{~s}$ before sensor 2 (see Fig. $5 \mathrm{D}$ left). In terms of all subjects, the firing rates before sensor 1 are significantly higher than those before sensor 2 (see Fig. 5D right, Wilcoxon signed-rank test, $p<0.05$ ). These results further suggest that the NCL neural activities may be related to the decision-making during goal-directed navigation tasks.

\subsection{NCL decoding performance during the decision-making}

If the NCL neural activities contain the decision-making information, the signals during the decision-making should be capable of decoding animals' movement direction. A sliding decoding analysis was performed on a larger window, $5 \mathrm{~s}$ centered on each sensor (see Fig. 6A). These windows were defined as goS1, goS2, returnS2, and returnS1, respectively. An example of a spike train for a channel in these windows is shown in Fig. 6B. The results have shown that the firing rates are different in the four windows. The firing rates are higher than those in other windows for $2.5 \mathrm{~s}$ after sensor 2 in the goS2 window and $2.5 \mathrm{~s}$ before sensor 2 in the returnS2 window. More importantly, the firing rates are different for different movement directions. The results have shown that the NCL neural activities during the decision-making can decode the movement direction of pigeons.

To verify the conclusion mentioned above, a sliding window of $1 \mathrm{~s}$ width and steps of $0.05 \mathrm{~s}$ was used for decoding. The result revealed that the decoding accuracy increases significantly near sensor 2 in the going and sensor 1 in the returning (see Fig. 6C). Moreover, regarding the goS2, the decoding accuracy after sensor 2 is significantly higher than that before sensor 2 (see Fig. 6D; Wilcoxon signed-rank test, $p<$ $0.05)$. For the returnS2, the decoding accuracy before sensor 2 is significantly higher than that after sensor 2 (Wilcoxon signed-rank test, $p<0.05)$. It is also significantly higher than the chance level $(\sim 0.33$; permutation test, $p<0.05)$. However, the decoding accuracy shows no significant differences between before and after sensor 1 for the other two windows (permutation test, $p>0.05$ ). Together, the results obtained verify the above conclusion again that the NCL neural activities may be related to the decision-making during the goaldirected navigation task. 


\section{Discussion}

In this paper, we explored the role of the avian NCL during the goal-directed navigation task. The results have shown that the NCL neural activities may be related to decisionmaking. The main findings are: (1) Neural activities obtained from the NCL may contain decision-making during the goaldirected navigation task. (2) The location of the decisionmaking is different between the random-goal task and the fixed-goal task. The decision-making window is at the intersection for the random-goal task and departure for the fixedgoal task. (3) The avian NCL may play a similar role with the mammalian PFC for goal-directed spatial decision-making. These results may provide some support for understanding the neural decision-making mechanism of birds.

The task for pigeons needs the subject to run from the starting location to the goal location as instructed by the food award. After the reward is consumed, it has to go back to the starting location. It is important to emphasize that, unlike previous maze tasks in which animals were trained to choose by a cue such as colors and tones, the subjects are cued by food and light located at the goal location. Thus, in the current task, going is a random-goal task, just like only knowing the rough location but failing to know where such as driving to find a restaurant in daily life. But the returning is a fix-goal task, such as going home after eating. The NCL neural responses at the intersection are completely different between the two tasks. This is consistent with our intuitive understanding. The decision-making window is at the intersection for the random-goal task, but it is a departure in the fixedgoal task. This difference reflects that the NCL neural activities are associated with the decision-making during the goaldirected navigation task.

Increasingly studies suggest that avian NCL as a convergence zone between the ascending sensory and the descending motor systems bridges the gap between stimulus delivery and behavioral execution $[12,22]$. In addition, our previous studies also have verified that the NCL neurons participate in the goal-directed navigation of pigeons [14], and it will receive the information input from the $\mathrm{Hp}$ area in the goal-directed decision-making [15]. The present data further supports the hypothesis that the NCL participates in goaldirected navigation. Additionally, its neural activities contain the decision-making information during goal-directed navigation. We designed two tasks and compared them horizontally and vertically to verify this conclusion. Based on the horizontal comparison, in the same task, the location of the NCL neural responses in the returning is significantly ahead of that in the going. From the vertical comparison, for the going in the different task, the location of neural responses in the fixed-goal task is significantly ahead of that in the random-goal task. As a result, these three processes together can make it highly likely that the NCL neural activities contain the decision-making information in the goal-directed navigation.
We have found that the firing rate in the going is higher than that in the returning. The peak of the firing rates is mainly distributed near sensor 2 rather than the center location of the intersection in the going for the random-goal task. This phenomenon is extremely interesting. It is well known that the reward plays a central role in goal-directed navigation. The reward-associated firing of NCL has been investigated in previous studies and will not be discussed here [14]. Therefore, we have believed that the role of dopamine, which is closely related to reward, maybe one of its potential reasons. Karakuyu et al. [9] have reported that dopamine has no unitary function but is differentially released during working and short-term memory in NCL. Moreover, Karakuyu et al. [9] has suggested that the efflux of the dopamine of NCL is related to short-term memory.

Puig et al. [23] have also reported that the birds have evolved a comparable dopaminergic system by comparing the role of dopamine transmission in the prefrontal cortex during learning and memory. The equipment and the function of NCL may also be the potential reasons. Hok et al. [6] have suggested that PFC neurons may be responsible for spatial goal coding in path planning. Due to NCL and PFC having similar functions, spatial coding is also one of the potential causes of the above phenomenon. However, the deep-seated reasons may need more in-depth research to reveal.

Our findings add to the growing body of evidence for the functional analogy between the avian NCL and the mammalian PFC. They are multimodal association areas that operate at the top of the telencephalic-processing hierarchy, ideally positioned to integrate sensory input and project to motor output. The PFC is thought to be critical for goaldirected behavior, and it is regarded as a necessity for guiding goal-directed navigation [3]. In the current work, we found that the NCL plays a critical role in goal-directed navigation, and its neural activities contain important information concerning goal-directed decision-making. The results have shown that both areas share a similar role in goal-directed navigation. Nevertheless, this similarity is surprising, considering the 300 million years of independent evolution and strikingly different neuro-architecture of the mammalian and avian brains [24].

\section{Conclusions}

To conclude, the current results promise much more than a mere demonstration that the NCL may contain the decision-making information during the goal-directed navigation task of pigeons. We have also found that the decisionmaking window differs between random and fixed-goal tasks. It is at the intersection for the random-goal task and departure for the fixed-goal task. This result verifies that the neural response of the NCL area is related to the decisionmaking activity of animals. Moreover, it provides new evidence for the functional homology between the avian NCL and the mammalian PFC. Together, these also support understanding the neural decision-making mechanism for dif- 
ferent species. Future experiments investigating the role of NCL in goal-directed navigation will further elucidate the possible neural information process mechanism for the birds.

\section{Abbreviations}

NCL, nidopallium caudolaterale; PFC, prefrontal cortex; $\mathrm{AP}$, anteroposterior; ML, mediolateral; DV, dorsoventral.

\section{Author contributions}

All authors listed have made a substantial contribution to the work. XYL, YY and KZ performed the experiments. XYL and $\mathrm{KZ}$ designed the experiments. XYL, YNP and HX analyzed the data and wrote the manuscript. DYW supervised the experiments. XYL and YY contributed equally to this work.

\section{Ethics approval and consent to participate}

All experiments were conducted in accordance with the Animals Act, 2006 (China) for the care and use of laboratory animals and approved by Life Science Ethical Review Committee of Huanghuai University.

\section{Acknowledgment}

We thank two anonymous reviewers for excellent criticism of the article.

\section{Funding}

This research was funded by National Natural Science Foundation of China, grant number 62003146; Key Scientific Research Projects of Colleges and Universities in Henan Province, grant number 21A413004; Major Commission Project of Industrial Innovation and Development Research in Zhumadian City, grant number 2020ZDWT08 and Young Teacher Foundation of Huanghuai University.

\section{Conflict of interest}

The authors declare no conflict of interest.

\section{References}

[1] Ito HT, Zhang S, Witter MP, Moser EI, Moser M. A prefrontalthalamo-hippocampal circuit for goal-directed spatial navigation. Nature. 2015; 522: 50-55.

[2] Penner MR, Mizumori SJY. Neural systems analysis of decision making during goal-directed navigation. Progress in Neurobiology. 2012; 96: 96-135.

[3] Churchwell JC, Morris AM, Musso ND, Kesner RP. Prefrontal and hippocampal contributions to encoding and retrieval of spatial memory. Neurobiology of Learning and Memory. 2010; 93: 415-421.

[4] Poucet B, Hok V. Remembering goal locations. Current Opinion in Behavioral Sciences. 2017; 17: 51-56.
[5] Ito HT. Prefrontal-hippocampal interactions for spatial navigation. Neuroscience Research. 2018; 129: 2-7.

[6] Hok V, Save E, Lenck-Santini PP, Poucet B. Coding for spatial goals in the prelimbic/infralimbic area of the rat frontal cortex. Proceedings of the National Academy of Sciences of the United States of America. 2015; 102: 4602-4607.

[7] Hok V, Chah E, Save E, Poucet B. Prefrontal cortex focally modulates hippocampal place cell firing patterns. The Journal of Neuroscience. 2013; 33: 3443-3451.

[8] Güntürkün O, Bugnyar T. Cognition without Cortex. Trends in Cognitive Sciences. 2016; 20: 291-303.

[9] Karakuyu D, Herold C, Güntürkün O, Diekamp B. Differential increase of extracellular dopamine and serotonin in the 'prefrontal cortex' and striatum of pigeons during working memory. The European Journal of Neuroscience. 2007; 26: 2293-2302.

[10] Güntürkün O. Cognitive impairments after lesions of the neostriatum caudolaterale and its thalamic afferent in pigeons: functional similarities to the mammalian prefrontal system? Journal Fur Hirnforschung. 1997; 38: 133-143.

[11] Diekamp B, Gagliardo A, Güntürkün O. Nonspatial and subdivision-specific working memory deficits after selective lesions of the avian prefrontal cortex. The Journal of Neuroscience. 2002; 22: 9573-9580.

[12] Güntürkün O. The avian 'prefrontal cortex' and cognition. Current Opinion in Neurobiology. 2006; 15: 686-693.

[13] Kirsch JA, Vlachos I, Hausmann M, Rose J, Yim MY, Aertsen A, et al. Neuronal encoding of meaning: establishing categoryselective response patterns in the avian 'prefrontal cortex'. Behavioural Brain Research. 2009; 198: 214-223.

[14] Liu X, Wan H, Li S, Shang Z, Shi L. The role of nidopallium caudolaterale in the goal-directed behavior of pigeons. Behavioural Brain Research. 2017; 326: 112-120.

[15] Zhao K, Nie J, Yang L, Liu X, Shang Z, Wan H. Hippocampusnidopallium caudolaterale interactions exist in the goal-directed behavior of pigeon. Brain Research Bulletin. 2019; 153: 257-265.

[16] Karten HJ, Hodos W. A stereotaxic atlas of the brain of the pigeon (Columba Livia). American Journal of Psychology. 1967; 81: 1-7.

[17] Paralikar KJ, Rao CR, Clement RS. New approaches to eliminating common-noise artifacts in recordings from intracortical microelectrode arrays: inter-electrode correlation and virtual referencing. Journal of Neuroscience Methods. 2009; 181: 27-35.

[18] Efron B. Bootstrap Methods: Another Look at the Jackknife. Springer: New York. 1979.

[19] Liu X, Ping Y, Wang D, Yao R, Wan H. Comparison of decoding performance between spike and local field potential signals during goal-directed decision-making task of pigeons. Journal of Biomedical Engineering. 2018; 35: 786-793.

[20] Anaene Oyeka IC, Ebuh GU. Modified Wilcoxon Signed-Rank Test. Open Journal of Statistics. 2012; 02: 172-176.

[21] Ojala M, Garriga GC. Permutation Tests for Studying Classifier Performance. Journal of Machine Learning Research. 2010; 11: 1833-1863.

[22] Güntürkün O. The convergent evolution of neural substrates for cognition. Psychological Research. 2012; 76: 212-219.

[23] Puig MV, Rose J, Schmidt R, Freund N. Dopamine modulation of learning and memory in the prefrontal cortex: insights from studies in primates, rodents, and birds. Frontiers in Neural Circuits. 2014; 8: 93.

[24] Veit L, Nieder A. Abstract rule neurons in the endbrain support intelligent behaviour in corvid songbirds. Nature Communications. 2013; 4: 2878. 